\title{
ANÁLISIS DEL OBJETO ARQUITECTÓNICO DESDE LA TRANSPOSICIÓN TECNOLÓGICA. CASO: DOMO DEL MILENIO
}

Daniel E. VEDOYA (1) / Emma S. PRAT (2)

devedoya@arnet.com.ar; emmasus@hotmail.com

(1) Profesor titular de Construcciones II y director del ITDAHu; (2) profesora titular de Estructuras III y subdirectora del ITDAHu. FAU-UNNE

\author{
Palabras Clave: Transposición tecnológica; procesos que \\ generan procesos. \\ Keywords: technological transposition, processes generating processes
}

\section{RESUMEN}

El eje central del presente trabajo de investigación es la construcción de un método que permita interpretar la obra arquitectónica con un enfoque diferente, incorporando conceptos tecnológicos en el abordaje de la esencia misma de la arquitectura, aplicando una herramienta de análisis inédita: la Transposición Tecnológica. Esta herramienta se toma como principal estrategia para el análisis de las manifestaciones arquitectónicas que conforman la unidad de análisis, el Domo del Milenio, y ha sido una preocupación especial describir el significado que adquiere en la elaboración de este trabajo.

\begin{abstract}
The central axis of this research is the construction of a method for interpreting the architectural work with a different approach, incorporating technological concepts in addressing the very essence of architecture, implementing a tool unpublished analysis: the Technological Transposition. This tool is taken as the main strategy for analysis of architectural forms that make up the unit of analysis, the Millennium Dome, and has been a special concern to describe the meaning acquired in the development of this work.
\end{abstract}




\section{OBJETIVO GENERAL}

Desarrollar una metodología para el análisis de la obra arquitectónica, que comprenda los mecanismos de transposición tecnológica junto con las categorías vitruvianas y los principios de la arquitectura sustentable.

\section{OBJETIVOS ESPECÍFICOS}

- Identificar las dimensiones del análisis para el estudio de la obra arquitectónica desde el punto de vista tecnológico.

- Caracterizar las variables y dimensiones correspondientes a dichas categorías.

- Definir los mecanismos de transposición tecnológica que se pueden utilizar en el método de análisis de la obra arquitectónica.

- Relacionar los mecanismos de transposición tecnológica pertinentes con las categorías de VITRUVIO y los principios de la arquitectura sustentable (VITRUVIO, ca. S. I d. C).

\section{Antecedentes del objeto arquitectónico (antes)}

El proyecto del Millennium Dome fue concebido originalmente como algo de menor escala, durante el gobierno conservador de John Major, como una celebración del Festival of Britain o Exposición Mundial para celebrar el tercer milenio. El gobierno laborista entrante elegido en 1997 con el mando de Tony Blair amplió considerablemente el tamaño, alcance y presupuesto del proyecto. También aumentaron considerablemente las expectativas de lo que sería organizado. Justo antes de su inauguración, Blair afirmó que el Millennium Dome sería "un triunfo de la confianza sobre el cinismo, la audacia sobre el conformismo, la excelencia sobre la mediocridad". En palabras del corresponsal de la BBC Robert Orchard:

"el Dome debe ser subrayado como un brillante logro del nuevo laborismo en el próximo programa electoral".

Sin embargo, antes de su apertura el Millenium Dome fue condenado en una diatriba de lain Sinclair, que predijo con exactitud el exagerado bombo, la afectada pose política y la desilusión final.

La obra fue concebida para recibir el nuevo milenio, por lo cual se le otorgó un carácter magnánimo y con tecnología de avanzada para posicionar a Inglaterra dentro del escenario mundial y proporcionarle modernidad. Es importante destacar que fue construida únicamente para la llegada del año 2000. La cúpula se levantó como parte de la regeneración de la península de Greenwich en Londres y se invirtieron en ella casi tres mil millones de euros. La atracción resultó ser un fracaso, y en la actualidad, aunque permanece cerrada, su mantenimiento cuesta cerca de cincuenta millones de euros.

\section{Materialización del objeto arquitectónico (durante)}

\section{Dimensiones e indicadores}

\section{Utilidad (Uso - Función - Confort)}

Para festejar el comienzo del tercer milenio, la ciudad de Londres organizó una gran exposición que se abrió al público el 1 de enero de 2000 y se clausuró el 31 de diciembre del mismo año. Para albergar la exposición se le encomendó al arquitecto Richard Rogers el diseño para la construcción de un pabellón que luego se conoció con el nombre de Millenium Dome (Domo del Milenio, Fig. 1). 


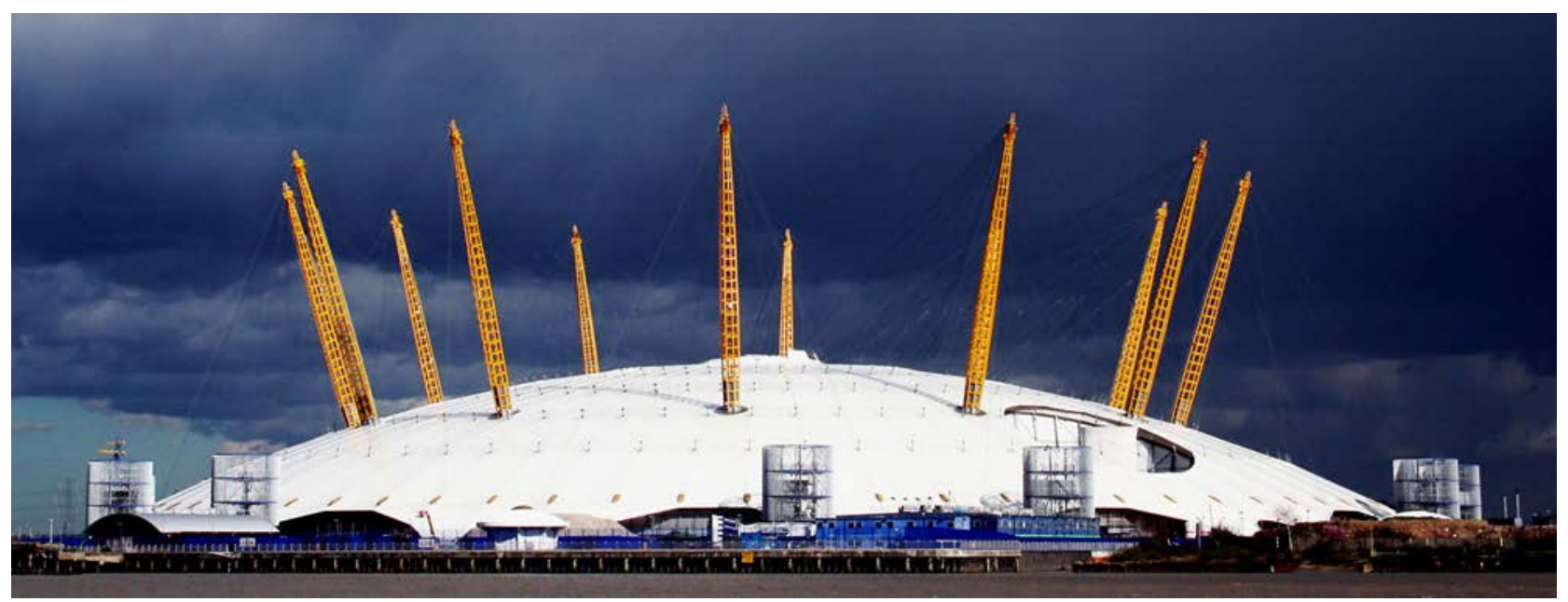

Fig. 1. Domo del Milenio, Londres. En su origen fue un centro de convenciones

El primer aspecto relevante es su ubicación geográfica, precisamente en el punto por donde pasa el meridiano de Greenwich, nombre de la península londinense que sirvió de asentamiento del domo, meridiano que señala la línea a partir de la cual se miden las longitudes terrestres. Por esta razón, también es conocido como meridiano cero, meridiano base o primer meridiano, y se corresponde con una circunferencia imaginaria que une los polos. Allí mismo se ubicaba antiguamente el antiguo observatorio astronómico que llevaba su nombre.

\section{Firmeza (Rigidez - Resistencia - Estabilidad)}

La planta circular presenta dos circunferencias concéntricas, una mayor que la otra. En la circunferencia menor se ubican doce columnas metálicas compuestas que soportan la estructura, apoyadas y articuladas sobre bases conformadas por cuatro tubos circulares que forman una base piramidal cuadrada de diez metros de altura, fundada en una base de hormigón (Fig. 2). Los tensores colaboran para generar la forma cupular, mantener en equilibrio el sistema y conferir al conjunto la necesaria rigidez y estabilidad (por eso son considerados estabilizadores del sistema estructural). Los superiores vinculan los cables de la membrana con los mástiles, transmitiéndole de esta manera el peso de la cubierta. De la parte superior de cada mástil salen veintiséis tensores de acero, de los cuales veintitrés se vinculan con los meridianos y paralelos, dos se anclan en un contrafuerte en el exterior de la membrana y uno sostiene el anillo central de la cúpula. Los tensores inferiores, que se hallan conectados a las bases de los mástiles, contrarrestan el efecto de succión del viento y le dan estabilidad al conjunto.

Todos los componentes del sistema (columnas, cables y membrana) están vinculados en puntos estratégicos (nudos) que posibilitan la resistencia

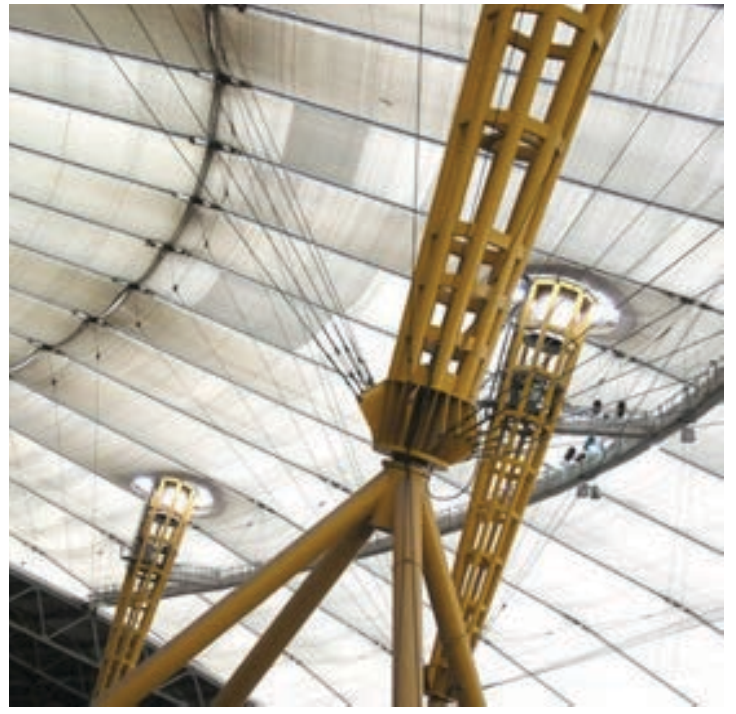

Fig. 2. Base piramidal de las columnas

global del conjunto. El nudo superior soporta, por medio de los tensores, el sistema de cables y la membrana superior. El nudo central soporta las tensiones generadas por los meridianos, que a su vez soportan los paralelos para formar el entramado. Se materializó por medio de una masa circular de acero, de la cual se desprenden bielas a las que van fijos los elementos de ensamble de los tensores. Al nudo inferior concurren los tensores inferiores. Los nudos son articulados, lo que determina un sistema isostático de tracción.

Cada mástil que soporta la cubierta está compuesto por ocho tubos estructurales vinculados entre sí por una unión que se llevó a cabo soldando un caño de sección rectangular entre los tubos circulares conformándose de esa manera una sección octogonal que le da un elevado momento de inercia. Una red de círculos concéntricos de cables de acero (paralelos) se entrelazan con otros radiales (meridianos) y constituyen la trama que 
da forma al conjunto y sobre la cual se apoya la membrana de fibra de vidrio recubierta con teflón (PTFE).

\section{Belleza \\ (Orden - Proporción - Ritmo)}

La cúpula simbolizaba un punto geográfico muy especial, dado que su ubicación marcaba precisamente el lugar por donde pasa el "axis mundi" (eje cósmico), el "omphalos" (ombligo del mundo) que une lo bajo con lo alto, el mundo subhumano con el suprahumano. La idea generadora parte de un globo terráqueo visto en planta, con sus respectivos paralelos y meridianos. Los componentes de la estructura se organizan en forma radial, distribuidos en ángulos de treinta grados.

Externamente se asemeja a una gran carpa blanca con doce torres amarillas de sujeción de cien metros de altura, una por cada mes del año (o también puede entenderse como correspondientes a cada hora de la esfera del reloj, representando el papel jugado por el tiempo medio de Greenwich). La planta del edificio es circular, de 365 m de diámetro, simboliza los días del año y ocupa 100.000 m2 de superficie, con una altura de cincuenta metros, con bordes ondulados.

Al igual que la arquitectura deconstructivista, el aspecto metafórico fue uno de los propósitos que guiaron la definición del espacio, tanto exterior como interior, y la distribución de sus componentes estructurales y funcionales. La distribución de las doce columnas representa, a su vez, tanto los doce meses del año como las doce horas del día, y se distancian una de la otra en treinta grados, completando en total los trescientos sesenta de la circunferencia de la planta. La circunferencia mayor, que determina el límite exterior de la cúpula, mide exactamente 365 metros de diámetro, y simboliza los días del año.

\section{Sustentabilidad (Ecología - Economía -Tecnología)}

El arquitecto Richard Rogers es uno de los defensores a ultranza de la arquitectura sustentable. Con su propio estudio, abierto en 1977, desarrolló edificios que llevan como bandera el empleo racional de la energía (para iluminación y climatización) y que pueden adaptarse a distintos usos, como el Consejo Lloyds en Londres, donde para lograr la flexibilidad genera espacios despejados mediante sistemas estructurales eficientes y que a su vez permiten la libre circulación del aire. El espacio interior está climatizado artificialmente. A la obra se accede peatonalmente desde un parque que incluye un sector de tratamiento orgánico de las aguas que logra un ambiente agradable, que se generó gracias a la recuperación de la zona que en su momento se encontraba contaminada.

En materia de energías renovables, el Domo del Milenio no es un modelo que hay que tomar. El consumo energético excesivo fue quizá uno de los temas que más rechazo obtuvo en relación con este proyecto. No obstante, el proyecto de remodelación propuesto para adaptar el espacio a conciertos y competencias deportivas, ejemplo más destacado, prevé una adecuación de los recursos energéticos, de modo de lograr criterios de ahorro más efectivos.

El HightTech es un movimiento arquitectónico que usa tecnologías propias de la producción industrial y la investigación aeroespacial. Richard Rogers predica que la tecnología no es un fin en sí mismo, sino un medio para solucionar problemas sociales y ecológicos, en contraste con otros arquitectos que proponen una arquitectura sofisticada basada en la tecnología. Otro de los objetivos perseguidos por el arquitecto es conseguir sistemas de construcción imaginativos y económicos. 
"Se presentan en la solución estructural del domo un variado número de contradicciones, resultado de sucesivas transposiciones tecnológicas..."

\section{RESULTADOS}

\section{Mecanismos de Transposición Tecnológica}

La obra fue concebida para recibir el nuevo milenio otorgándole un carácter magnificente, con tecnología de avanzada, con el objeto de brindarle a Inglaterra cierta dosis de modernidad y posicionarla dentro del escenario mundial emergente. "La actitud técnica desarrollada por el usuario de un determinado objeto es transferida a un nuevo objeto, con ciertas variantes con respecto a aquél. Estas variantes reemplazan las operaciones que motivaban aquella actitud" (primer mecanismo de Transposición Tecnológica. VEDOYA, 2014).

Se presentan en la solución estructural del domo un variado número de contradicciones, resultado de sucesivas transposiciones tecnológicas, como se verá en el transcurso del presente análisis. Una primera contradicción, que salta a la vista, es la columna o mástil que aparece como componente estructural, totalmente extraño en la conformación de las cúpulas (Fig. 3), correspondiente con el primer mecanismo de Transposición Tecnológica. Recuérdese que una cúpula es una superficie estructural rígida, cuyo comportamiento responde a la acción de las cargas de peso propio y

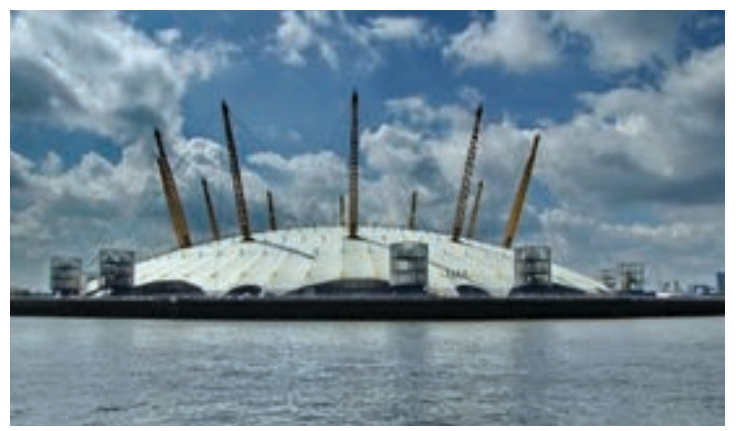

Fig. 3. Vista general del Domo donde se destacan las doce columnas sobrecarga. El equilibrio de un punto cualquiera de una cúpula rígida se logra gracias a la conjunción de fuerzas concurrentes: por una parte, la carga de peso propio y sobrecarga (vertical), que actúa sobre la estructura, y por otra, las resultantes de esta acción que se descompone en dos direcciones, una correspondiente a la curva que sigue el meridiano y la otra en sentido horizontal, correspondiente a cada paralelo (VEDOYA Y PRAT, 2009).

El plano neutro divide a la superficie en dos sectores, y su ubicación es constante para cada tipo de curva generatriz de la conformación de la cúpula (circunferencia, parábola, catenaria, etc.). La ubicación de este plano neutro se determina en la intersección de una línea ficticia con la superficie. La inclinación de esta línea ficticia con respecto al eje vertical varía según la curva generatriz de la superficie, pero es constante para cada tipo de curva. Así, para la circunferencia, curva generatriz de una esfera, su inclinación es de $51^{\circ}$ 49' (Fig. 4). En la intersección de esta línea con el plano medio de la esfera se localiza el punto por donde pasa el plano neutro. Esta posición es constante para la esfera, cualquiera sea su tamaño, y no depende de la distancia que haya desde su punto superior hasta la base. Esto quiere decir que, cualquiera sea la altura en que se corte a la cúpula con respecto a su plano medio, el plano neutro siempre se ubicará con respecto al centro de las coordenadas (Fig. 5).

La superficie resiste la acción de las cargas actuantes (peso propio más sobrecarga), soportando dos tipos de esfuerzos, uno de compresión, tangente a la curva de los meridianos, y el otro de compresión o de tracción, según se ubique sobre o debajo del plano neutro (Fig. 6). Cuando la base de la cúpula se sitúa por encima del plano neutro, los paralelos trabajan todos a la compresión, a excepción del paralelo situado en el borde de aquella, que bruscamente cambia el sentido de la acción y trabaja a tracción (Fig. 7). Este caso de cúpulas recibe el nombre de "cúpulas rebajadas". 


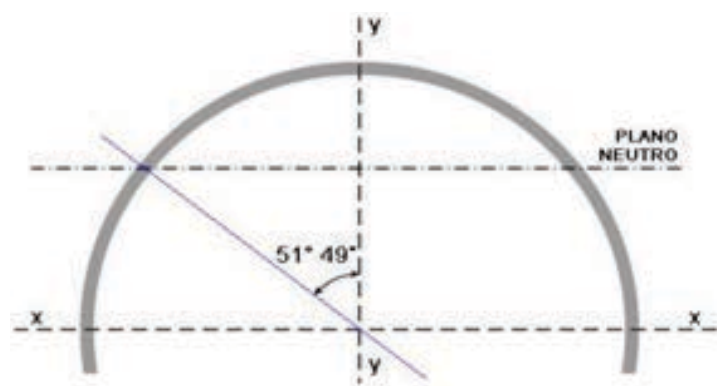

Fig. 4. Ubicación del plano neutro en una cúpula esférica

La explicación de este fenómeno lo da el principio de equilibrio que rige en toda estructura, representado por tres ecuaciones: la sumatoria de momentos igual a cero $(\Sigma M=0)$, la sumatoria de fuerzas verticales igual a cero $(\Sigma \mathrm{V}=0)$ y la sumatoria de fuerzas horizontales igual a cero $(\Sigma \mathrm{H}$ = 0). (VEDOYA Y PRAT, 2009).

La cúpula es una estructura superficial rígida que se comporta exclusivamente por la acción equilibrada de las fuerzas actuantes, no existiendo cargas excéntricas que pudieren generar momentos, porque estos, por la naturaleza misma de las cúpulas, son nulos, es decir, no existen en este tipo de estructuras. Las fuerzas verticales correspondientes a las cargas que actúan sobre la superficie se equilibran (se anulan) con la reacción que reciben del suelo. Las fuerzas horizontales (en el sentido de los paralelos) se complementan entre sí, equilibrándose las de compresión (por sobre el plano neutro) con las de tracción (por debajo del plano neutro).

¿Qué sucede, entonces, cuando la cúpula es rebajada? ¿No se produce aquí el equilibrio que anula la acción de las fuerzas horizontales? Lo que sucede es la acción de una fuerza horizontal que se localiza precisamente en el borde de la cúpula, que produce un cambio brusco de tensiones con consecuencias negativas para la estructura, ya que en ese sector se producen perturbaciones que derivan en fisuras y hasta en el colapso del sistema.

La solución que dan algunos autores es un engrosamiento de esta parte de la estructura, en el que colocan una adecuada armadura de tracción. NERVI, por su parte, encontró otra solución muy interesante colocando columnas inclinadas rodeando el Palazzetto dello Sport, en Roma (Fig. 8).

Reproducir en el Domo del Milenio la distribución de tensiones según las dos direcciones principales, siguiendo los lineamientos de una cúpula rígida,

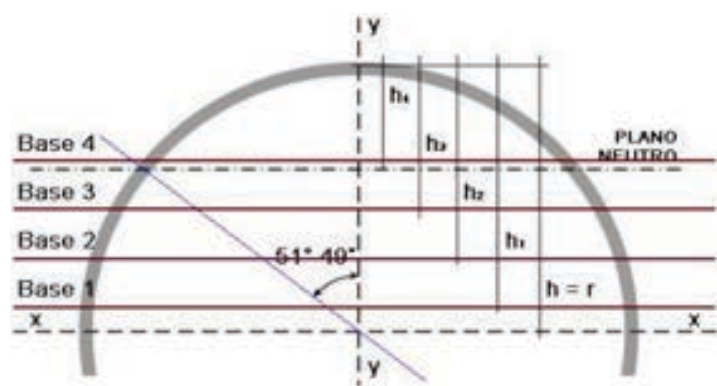

Fig. 5. Cualquiera sea la altura de la cúpula esférica, la ubicación del plano neutro es una constante

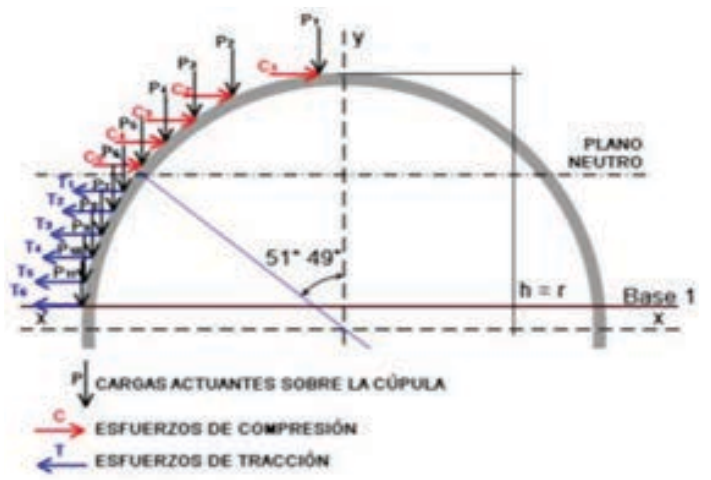

Fig. 6. Distribución de los esfuerzos en la cúpula

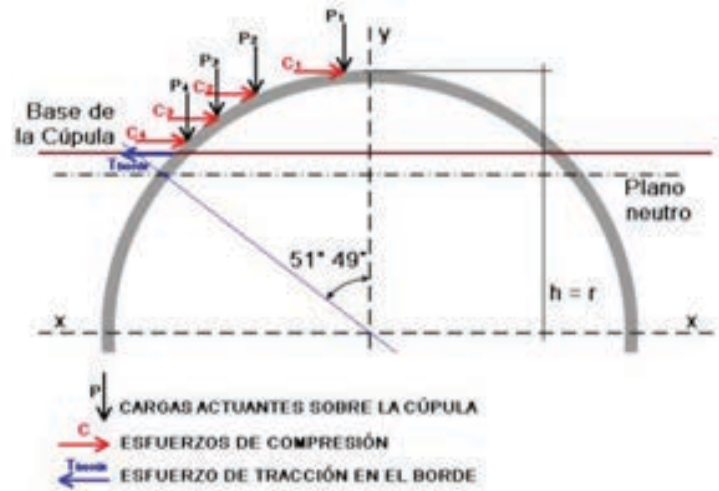

Fig. 7. Cúpula rebajada

aún no resuelve la cuestión. En la cúpula rígida, un punto cualquiera de ella se mantiene en equilibrio precisamente por esa cualidad de rigidez que presenta la estructura, es decir, el punto mantiene su posición en el espacio sin caer por efecto de la gravedad.

En el Domo del Milenio, por tratarse de una membrana flexible, esa situación no es posible. Es aquí donde se aplica el primer mecanismo de la Transposición Tecnológica, recurriendo al caso de las estructuras neumáticas. Aquí el equilibrio de un punto cualquiera de la membrana inflada se logra 


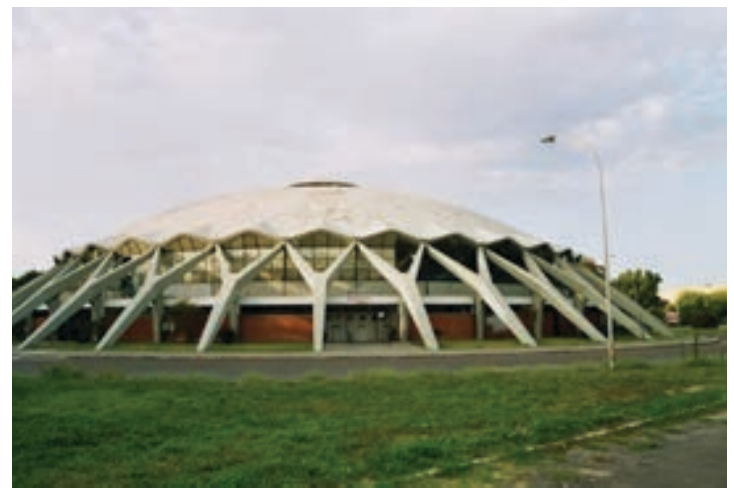

Fig. 8. Vista donde se aprecian las columnas inclinadas en el Palazzetto dello Sport, de Nervi

mediante la presión del aire interior del espacio, que en este caso debe ser cerrado totalmente para evitar pérdidas de presión (Fig. 9) (VEDOYA Y HERMIDA, 2009). No es este el caso del Domo del Milenio.

La solución es emular la acción de la presión debida al aire interior en la membrana inflada por algún mecanismo que impida que el punto considerado se precipite al suelo por efecto de la gravedad. Entonces aparecen los tensores que sustentan esos puntos evitando su caída, y las columnas o mástiles se hacen imprescindibles como elementos de sostén de cuyos extremos superiores cuelgan dichos tensores (Fig. 10).

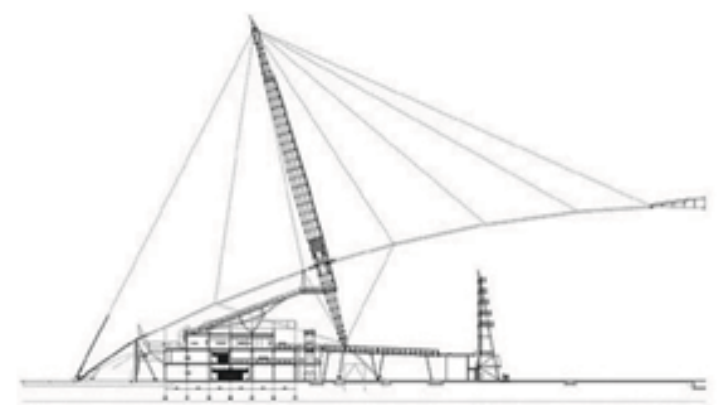

Fig. 10. Los tensores que sustentan la membrana están vinculados con la columna.

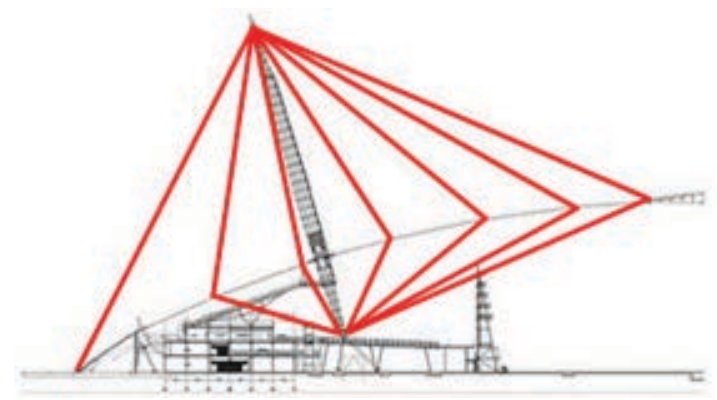

Fig. 11. Detalle del conjunto de tensores vinculados con la membrana.

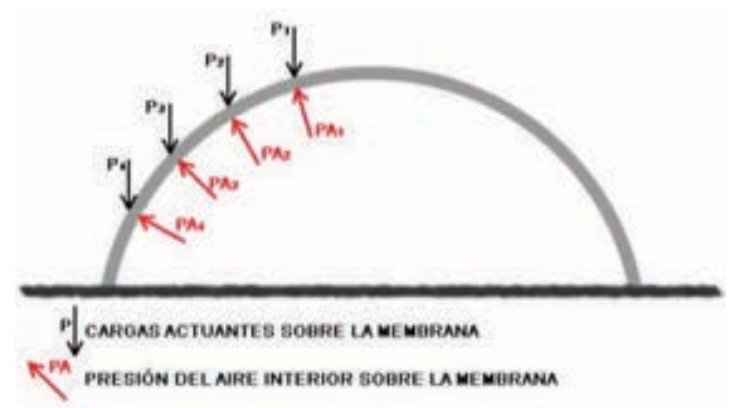

Fig. 9. Detalle del equilibrio de un punto cualquiera en una membrana inflada

Todavía falta resolver otra cuestión. Se ha logrado evitar que el domo se precipite a tierra gracias a los tensores que cuelgan de las columnas y sostienen la membrana. Pero ¿cómo se comportará la membrana si es sometida a la acción del viento? Se ha logrado evitar que caiga, pero no el riesgo de flameo por la acción del viento. Nuevamente, el primer mecanismo de la Transposición Tecnológica permite resolver la cuestión. Nuevos tensores aparecen en el sistema estructural, aunque ahora su función ya no es sostener la membrana por arriba, sino que están prendidos de esta en los mismos puntos en que se conecta con los tensores que cuelgan desde las columnas, y vinculan la membrana con la base misma de las columnas, formando una especie de trama espacial cónica que contrarresta cualquier acción que pueda provenir del viento (Fig. 11).

"El cambio que se produce en un objeto implica una simplificación de la actitud técnica del usuario para su empleo. Se produce una mayor complejidad interna en los procesos de funcionamiento del objeto (mecanismo de 'caja negra'). Esta simplificación puede referirse a una menor atención en los procedimientos de empleo, y también en una reducción del número de tareas necesarias para su uso" (segundo mecanismo de Transposición Tecnológica. VEDOYA, 2014).

El diseñador de estructuras ni siquiera tiene en cuenta todas estas reflexiones acerca del comportamiento de una cúpula. Es así como debe ser, y esto es suficiente para seguir adelante con el diseño. La actitud del diseñador estructural se basa ciertamente en una verdadera transposición tecnológica, que lo hace actuar sin siquiera pensar que lo está haciendo. Porque todo esto que se ha dicho queda enquistado en lo que se conoce como la "caja negra" del diseño, que no es necesario conocer (segundo mecanismo de Transposición Tecnológica), solo se requiere tener la certeza de que se está obrando conforme las normas que rigen 
la materia. Es como accionar un picaporte. Se sabe que accionando el picaporte se logrará correr el pestillo que traba la puerta. Al actor, en este caso, no le interesa saber lo que pasa en el interior de la cerradura; tampoco le resulta necesario saberlo. Aquí pasa lo mismo, solo basta con decidir qué se quiere conseguir y conocer cuáles son las acciones que se deben realizar para lograrlo.

Se sabe que una cúpula se comporta de determinada manera porque ya, a su tiempo, especialistas y expertos en la materia han estudiado el problema y llegaron a esta conclusión que con el tiempo se convirtió en premisa. Volviendo al momento en que se comenzó el análisis de cómo se mantiene en equilibrio un punto cualquiera de la cúpula, cabe recordar que se estaba hablando de una estructura superficial rígida. La noción de pandeo es la aplicación del segundo mecanismo de Transposición Tecnológica, con el cual se obvia todo el proceso de análisis de una pieza estructural cuya función es la de una columna sometida a la acción de una carga axial que podría producirle un efecto de pandeo. Se sabe cuál es la causa que produce el pandeo y se conocen los resultados si se aplican los coeficientes que alguien (experto, especialista, estudioso de los fenómenos estructurales) resolvió a su tiempo. Ya no interesa conocer más detalles, porque todo se condensa en el mecanismo de "caja negra".

Se sabe que hay que reducir la "altura de pandeo" de la pieza estructural, y debido a ello las columnas o mástiles del Domo del Milenio han sido diseñadas sectorizadas mediante caños de sección cuadrada soldados. La inercia de la columna requiere determinada sección en la zona media de su altura, pero no así en los extremos. Esto llevó al diseñador a resolver la cuestión otorgándole al mástil un perfil ahusado, descomponiendo la sección necesaria en ocho tubos curvados, que van separándose a medida que avanzan hacia la zona media de la columna, de cien metros de longitud total. "La integración de dos o más procesos diferentes da por resultado la producción de nuevos procesos" (quinto mecanismo de Transposición Tecnológica. VEDOYA, 2014).

La solución práctica del caso del Domo del Milenio está dada por el quinto mecanismo de Transposición Tecnológica, por la conjunción de dos tecnologías diferentes y antagónicas, una debida a la acción de fuerzas concurrentes (carga actuante y esfuerzos en el meridiano y el paralelo), equilibradas gracias a la rigidez del sistema, la otra por el principio de acción y reacción de dos fuerzas concurrentes opuestas (carga actuante y presión del aire interior).

Rogers resolvió esta cuestión conteniendo las tensiones en la membrana mediante una trama de cables que recorren la curvatura del domo según las dos direcciones principales: la de los meridianos y la de los paralelos. Resuelto el equilibrio de la membrana flexible del Domo del Milenio, resta ahora analizar el diseño de la columna o mástil.

Cada mástil está compuesto por ocho tubos estructurales vinculados entre sí por una unión que se llevó a cabo soldando un caño de sección rectangular entre los tubos circulares que conforma de esa manera una sección octogonal que le da un elevado momento de inercia, a lo que se suma un ensanchamiento de casi cuatro metros en su zona central, que reduce el efecto de pandeo que de otro modo pondría en riesgo la columna de cien metros de altura (Fig. 12).

"Los atributos de un proceso (su organización interna, su estructura, las interrelaciones entre sus componentes, su comportamiento) se reproducen en procesos análogos. No existe entre ellos una íntima relación, ni en sus componentes, ni en su función específica" (sexto mecanismo de Transposición Tecnológica. VEDOYA, 2014). 


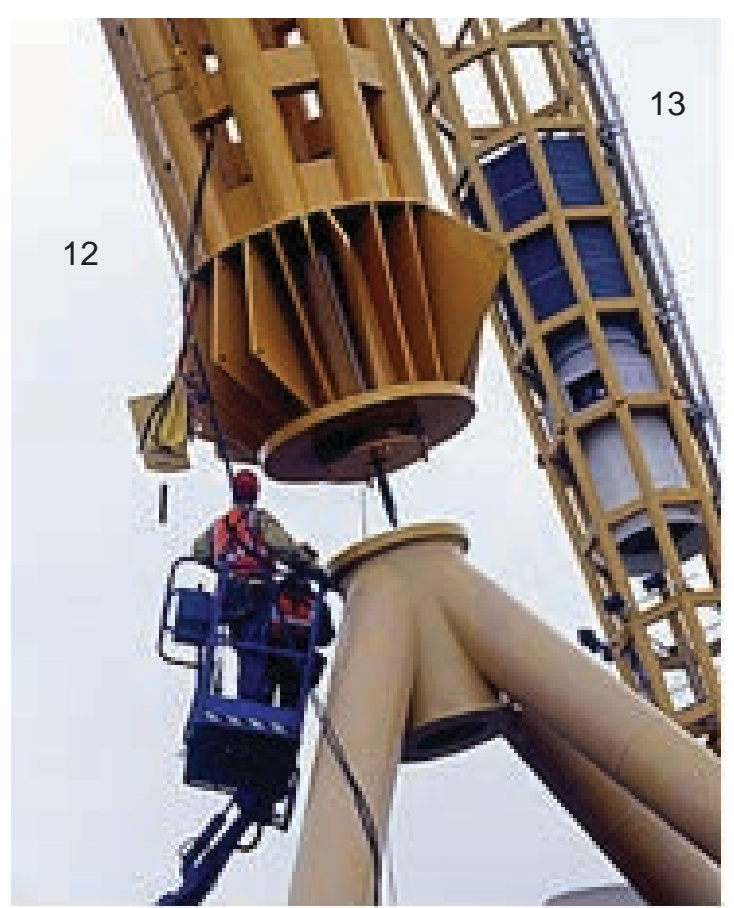

Figs. 12 y 13. Vista de la columna compuesta. Detalle de la columna compuesta

Los bordes del domo son ondulados (Fig. 14) y hacen recordar, por su forma, aunque no por su comportamiento estructural, a las columnas inclinadas que soportan el Palazzetto dello Sport (Fig. 15), del ingeniero PIER LUIGI NERVI, en Roma (sexto mecanismo de T. T.). "Los atributos de un proceso (su organización interna, su estructura, las interrelaciones entre sus componentes, su comportamiento) se reproducen en procesos inversos. Tampoco existe entre ellos una íntima relación, ni en sus componentes, ni en su función específica. Lo que en el proceso inicial constituía la causa, en el nuevo proceso se convierte en el resultado, y viceversa, lo que antes era el resultado, ahora es la causa" (séptimo mecanismo de Transposición Tecnológica. VEDOYA, 2014).

No obstante, la cúpula rígida no es el único modo de resolver esta forma. También hay ejemplos de cúpulas flexibles, aunque en este caso la solución es inversa (séptimo mecanismo de T. T.), y la solución de equilibrio se logra mediante la presión del aire interior del ambiente que acciona en sentido contrario a la gravedad, tratando de expandir la membrana flexible. Es el caso de las estructuras infladas o neumáticas, donde la membrana se opone a la acción de la presión del aire interior y de este modo se logra el equilibrio del conjunto. Acá el peso propio de la cubierta se equilibra con una fuerza de sentido contrario (en sentido contrario a la gravedad), producida por la presión del aire contenido en el interior de la cúpula. Lógicamente, este tipo de solución exige cierta hermeticidad del conjunto para evitar la pérdida de presión.

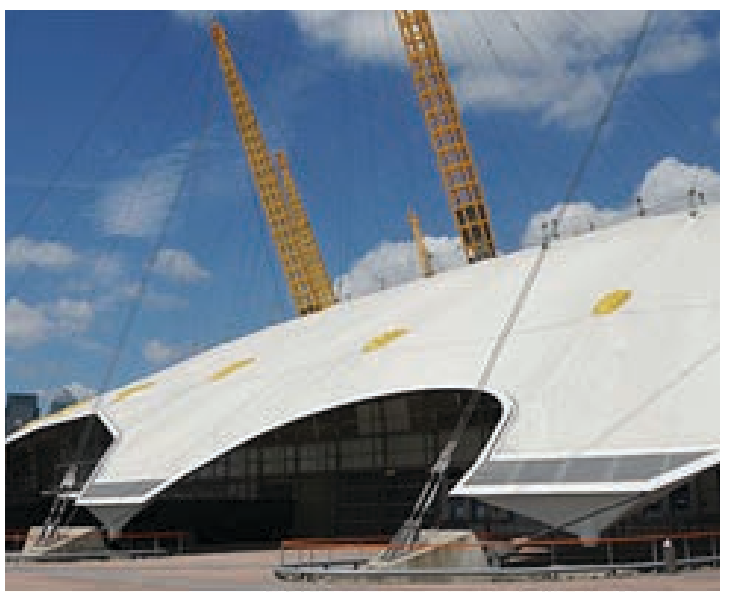

Fig. 14. Borde de la cubierta del domo

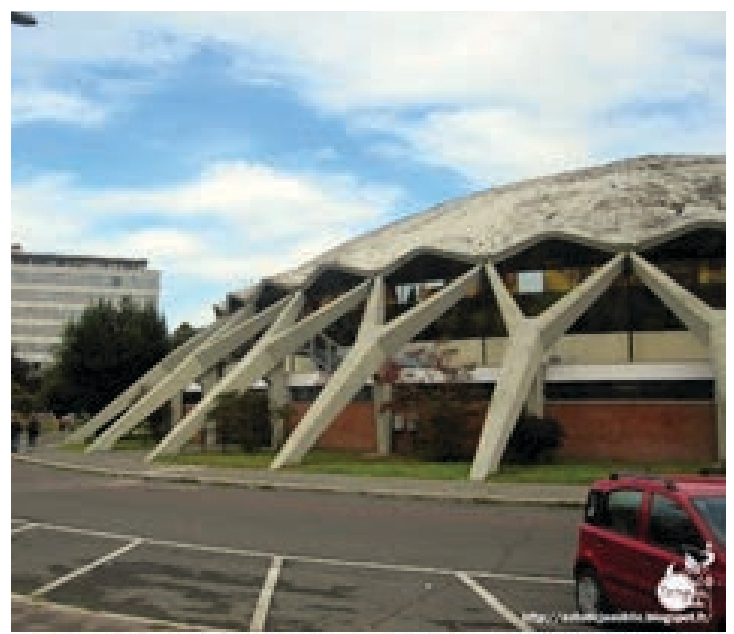

Fig. 15. Columnas inclinadas en el Palazzetto 
Al contrario de estas observaciones, el Domo del Milenio es una estructura conformada por elementos no rígidos, una membrana de fibra de vidrio recubierta con teflón (PTFE), cables de acero y columnas compuestas. Visto así, no se encuadra en absoluto en las características expuestas.

¿Cómo mantener entonces el equilibrio de un punto cualquiera de la superficie? Está claro que no se puede partir del análisis hecho precedentemente. $\mathrm{Si}$ bien se tiene un punto en equilibrio en un determinado sector de la superficie, esto no se debe ni a la rigidez de la estructura (porque no existe rigidez propia del material, que es una membrana flexible), ni a determinada presión del aire interior, dado que no se trata de un espacio cerrado hermético, sino de un ámbito abierto por sus lados.

Como se dijo, la cúpula del Milenio es una superficie no rígida, resuelta con una membrana flexible de fibra de vidrio recubierta con teflón (PTFE). Esta situación contradice el principio de rigidez de una cúpula, pues la membrana no está capacitada para resistir esfuerzos de compresión y sí solo los de tracción. Por esta razón, a pesar de que la forma de la estructura es la de una cúpula, esta no trabaja como tal, y requiere algunos recursos extraños a la esencia de una cúpula para mantener en equilibrio un punto cualquiera de ella. Estos recursos extraños se refieren a mecanismos que emulen el comportamiento de la cúpula, sea esta de material rígido o se trate de una membrana flexible inflada.

Se destaca en el Domo del Milenio la presencia de mástiles (o columnas), de cuyos extremos cuelgan cables o tensores que soportan la cubierta, conjuntamente con otros cables o tensores interiores, vinculados con la base de las columnas, y una red de círculos concéntricos de cables de acero (paralelos), entrelazados con otros radiales (meridianos), que constituyen una trama que permite mantener en su sitio los puntos estratégicos que conforman la cúpula (Fig. 16 y 17).
En una cúpula rígida, el comportamiento estructural se basa en la distribución de las tensiones internas en el material según dos direcciones principales: la de los meridianos y la de los paralelos.

En la Fig. 18 se expone una síntesis de la relación entre las dimensiones e indicadores del Domo del Milenio, en Londres, y los mecanismos de transposición tecnológica que han participado en su diseño.

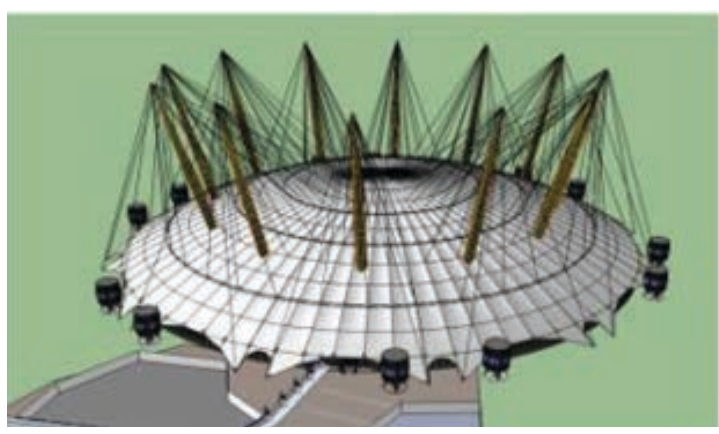

Fig. 16. Vista del conjunto de columna, tensores y cables paralelos

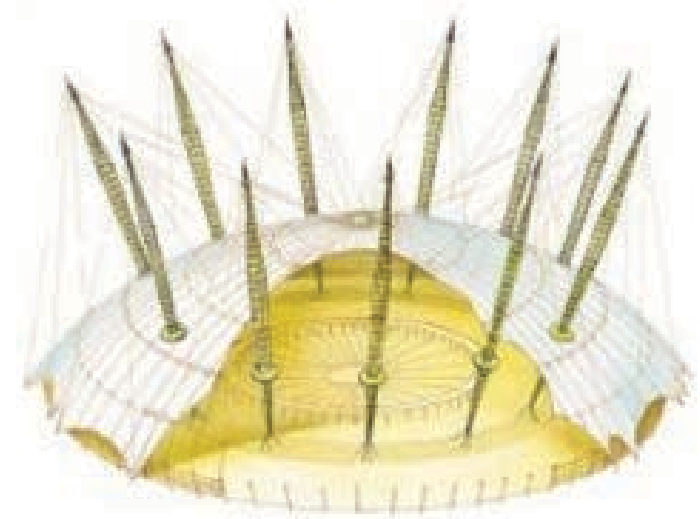

Fig. 17. Corte del conjunto de columna, tensores y meridianos y cables paralelos y meridianos 


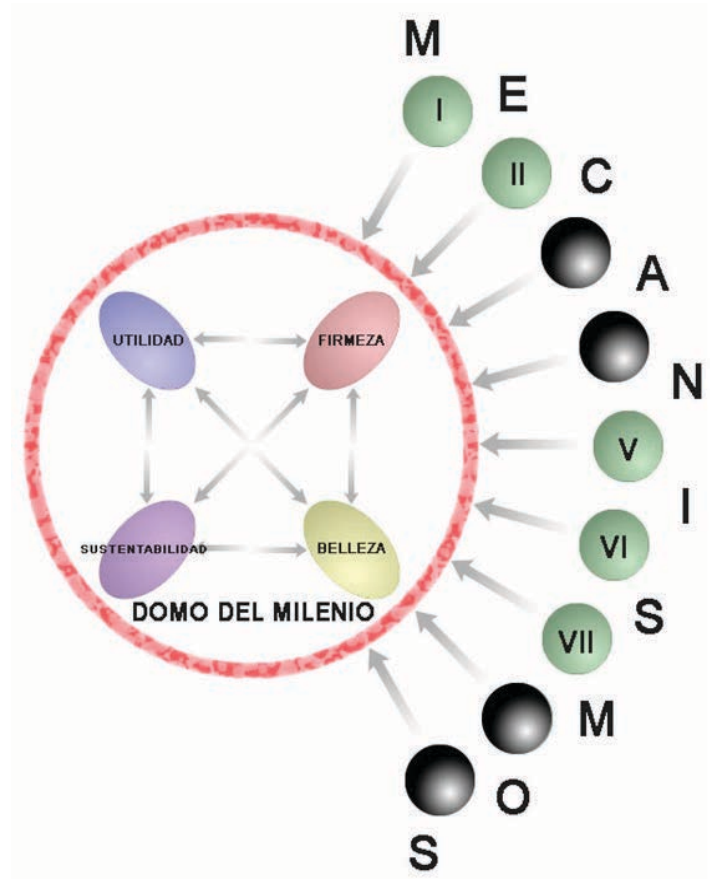

Fig. 18. Dimensiones de las variables e indicadores y mecanismos de Transposición Tecnológica que intervienen en la obra analizada dentro del contexto "Estructuras de Grandes Luces"

\section{CONCLUSIONES}

\section{Impactos del objeto arquitectónico (después)}

La existencia del Domo del Milenio fue efímera. Su reapertura se produjo a principios del año 2007 , después de una inversión de quinientos millones de libras esterlinas para transformar el espacio en un lugar de vanguardia destinado a conciertos y deportes. Desde la clausura de la exposición original se han propuesto y rechazado varias posibles formas de reutilizar el edificio. El cambio de nombre del domo el 31 de mayo de 2005 dio publicidad a la transición del Millennium Dome como zona deportiva cubierta. En este papel se pensó en albergar el Campeonato Mundial de Gimnasia de 2009, la final del circuito profesional de tenis ATP entre 2009 y 2012 y las competiciones de gimnasia artística y baloncesto de los Juegos Olímpicos de Londres 2012, así como dos partidos de la Liga Nacional de Hockey en 2007. La zona deportiva se complementa con un importante distrito de ocio.

También se ha planeado que sea el escenario de conciertos de figuras del panorama musical. De hecho, en 2007 la banda de rock Bon Jovi tuvo el privilegio de ser el primer grupo musical en tocar en dicho recinto, en un concierto en el que hubo lleno absoluto.
BIBLIOGRAFÍA

VEDOYA, Daniel Edgardo y HERMIDA, María del Carmen (2009) Principios básicos para la estructuración del espacio. Ediciones del ITDAHu, Corrientes, Argentina.

VEDOYA, Daniel Edgardo y PRAT, Emma Susana (2009) Estructuras de grandes luces. Tecnología y Diseño. Ediciones del ITDAHu, Corrientes, Argentina.

VEDOYA, Daniel Edgardo (2014) La transposición tecnológica. Introducción a la génesis de los procesos tecnológicos - Editorial Académica Española, Saarbrücken (Alemania).

VEDOYA, Daniel Edgardo (2014) La transposición tecnológica. Una estrategia para el diseño y análisis de la obra arquitectónica con en foque tecnológico. Editorial Publicia, Saarbrücken, Alemania.

VITRUVIO POLIÓN, Marco Lucio (ca. S. I d.C.) (1997) Los diez libros de la Arquitectura. Editorial Iberia SA, Barcelona, España.

Fuentes fotográficas

Figura 1: http://openbuildings.com/buildings/the-o2-arenaprofile- 1415

Figura 2: http://www.lusas.com/case/civil/millennium_dome.html Figura 3: http://www. panoramio.com/photo/22163439

Figura 8: http://www.epdlp.com/edificio.php?id=566

Figura 12: http://www.constructionphotography.com/

Figura 13: http://www.constructionphotography.com/

Figura 14: http://www.urban75.org/london/the-02-millennium-dome htm

Figura 15: http://astudejaoublie.blogspot.com.ar/2013/12/rome-palaisdes-sports-village.html.

Figura 16: ARQHYS Arquitectura.

Figura 17: ARQHYS Arquitectura. 